\title{
Numerical Simulation of Friction Stir Welding of Magnesium Alloy considering Fluid Dynamics
}

\author{
Moosun Kim¹, Jung-Seok Kim² \\ ${ }^{1}$ Metropolitan Transit Convergence Research Division, Korea Railroad Research Institute \\ \#176 Cheoldo bakmulkwan-ro, Uiwang-si, Gyeonggi-do, South Korea \\ ${ }^{2}$ Advanced Materials Research Team, Korea Railroad Research Institute \\ \#176 Cheoldo bakmulkwan-ro, Uiwang-si, Gyeonggi-do, South Korea \\ mskim@krri.re.kr; jskim@krri.re.kr
}

\section{Extended Abstract}

Friction stir welding process is considered as an eco-friendly technology because it does not generate arc and harmful gas during process compared to the conventional welding process. For this reason, it is mainly applied to manufacturing of transportation such as marine, aviation, automobile and railway. Most of researches on friction stir welding has considered aluminum alloy so far and researches on the application of magnesium alloy, which is attracting attention as a lightweight material, is insufficient. Therefore, it is necessary to study the phenomenon of the friction stir welding of magnesium alloy.

Friction stir welding is a solid-state jointing process in which welding is performed using a frictional heat generated between the metal materials and tools by high-speed rotation of a welding tool. The main criterion for evaluating the quality of the friction stir welding is determined by the magnitude of the heat input generated by friction, and problems such as oxide or tunnel defects in the material occur due to the excessive or insufficient heat input. Therefore, it is very important to grasp the temperature history of the material during process for the successful welding result. As a method to predict the temperature history under the various welding conditions, there is a structural analysis approach considering the plastic deformation of material and a flow analysis approach considering the welding material as a high viscous fluid.

In this study, the flow velocity and temperature of material in the friction stir welding process were numerically analysed considering fluid dynamics. Magnesium alloy AZ31 was considered as the welding material and assumed to be non-Newtonian fluid. Fluent was used as a commercial computational fluid dynamics program for numerical analysis. As the variables for analysis, the rotational speed of the welding tool, the feed speed of the welding base material, and the shape of the welding tool were considered. For the verification of the numerical analysis results, the temperature measuring test inside the welding region of the magnesium alloy was performed. By comparing results, it was confirmed that the temperature profiles agreed well.

This research was supported by a grant from R\&D Program of the Korea Railroad Research Institute, Republic of Kor ea

\section{References}

[1] S. Sun, J. Kim and W. Lee, "Evaluation on temperature of FSW zone of magnesium alloy using experiment and FE analysis," Journal of the Korea Academia-Industrial cooperation Society, vol. 17, no. 7, pp. 434-441, 2016.

[2] S. Kang, B. Jang and H. Song, "Residual stresses analysis of friction stir welding using one-way FSI simulation," Journal of Mechanical Science and Technology, vol.29, no.3, pp.1111-1121, 2015.

[3] S. D. Ji, Q. Y. Shi, L. G. Zhang, A. L. Zou, S. S. Gao and L. V. Zan, "Numerical simulation of material flow behaviour of friction stir welding influenced by rotational tool geometry," Computational Materials Science, vol. 63, pp. 218-226, 2012. 